Little P, Stuart B, Moore M, et al. Amoxicillin for acute lower-respiratory-tract infection in primary care when pneumonia is not suspected: a 12-country, randomised, placebo-controlled trial. Lancet Infect Dis 2013; 13: 123-129.

7 British Thoracic Society. BTS guidelines for the management of community acquired pneumonia in adults. Thorax 2001; 56: Suppl. 4, iv1-iv64.

8 Watson L, Little P, Moore M, et al. Validation study of a diary for use in acute lower respiratory tract infection. Fam Pract 2001; 18: 553-554.

9 van Vugt SF, Broekhuizen BD, Lammens C, et al. Use of serum C reactive protein and procalcitonin concentrations in addition to symptoms and signs to predict pneumonia in patients presenting to primary care with acute cough: diagnostic study. BMJ 2013; 346: f2450.

10 Pakhale S, Mulpuru S, Verheij TJM, et al. Antibiotics for community-acquired pneumonia in adult outpatients. Cochrane Database Syst Rev 2014; 10: CD002109.

\title{
Tuberculosis screening using ability to provide sputum in an endemic emergency department
}

To the Editor:

Tuberculosis causes an enormous burden of morbidity and mortality worldwide [1]. Increasing rates of multidrug-resistant (MDR) and extensively drug-resistant (XDR) tuberculosis pose a serious threat to ongoing efforts in controlling the epidemic, particularly in low- and middle-income countries [2].

The World Health Organization (WHO) recommends tuberculosis screening via sputum smear examination in adults attending outpatient health facilities who report cough for $\geqslant 2$ weeks [3]. Little is known about optimal case finding definitions in emergency departments where the prevalence of tuberculosis appears to be higher than outpatient clinics [4]. Furthermore, emergency departments are highly important congregate settings bringing together the sickest diagnosed and undiagnosed tuberculosis patients with a vulnerable population, and may be the main portal of entry to care for persons who otherwise would not seek routine medical attention in outpatient clinics.

We assessed the use of a simple case finding strategy based on obtaining a sputum sample for acid-fast smear and culture in all emergency department comers who could produce a sputum sample, regardless of reason for emergency department visit, report of cough symptoms or cough duration.

The study was conducted between July 2005 and August 2006 in Hospital Nacional Dos de Mayo, a large public hospital in Lima, Peru, serving a low-income area with a tuberculosis rate of 113 cases per 100000 inhabitants [5]. Patients evaluated in the emergency department were invited to participate in the study if they met the following criteria: $\geqslant 18$ years of age, $\geqslant 2 \mathrm{~h}$ stay in the emergency department, and ability to provide an adequate sputum sample for acid-fast bacilli (AFB) smear and culture. Patients who were receiving tuberculosis treatment at enrollment were excluded. Patients were enrolled from 08:00 h to 20:00 h on Monday to Saturday. Participants provided written informed consent and were administered a standardised questionnaire to collect sociodemographic and clinical data including age, gender, residence location, occupation, medical history, tuberculosis risk factors and symptoms. One sputum sample was collected per patient.

Ziehl Neelsen AFB smear microscopy was performed on all sputum samples at the hospital laboratory. Sputum samples were cultured on acid-buffered Ogawa media for 8 weeks [6]. Susceptibility testing was performed at the Lima-Ciudad Regional Laboratory using the proportional method of CANETTI and GROSSET [7].

Pulmonary tuberculosis (PTB) was defined as having a sputum culture positive for Mycobacterium tuberculosis. MDR-tuberculosis was defined as cultures resistant to at least isoniazid and rifampin. PTB patients were offered counselling for HIV testing. Stata software (version 12.0; StataCorp, Texas) was used for analysis and a p-value $\leqslant 0.05$ was considered significant. The study was approved by the hospital ethics committee.

Over the study period, 931 patients were enrolled. Of these, 174 (18.7\%) had PTB. Table 1 shows the characteristics of PTB patients by cough report. $42(24.1 \%)$ of the 174 PTB patients reported no cough. 26 (14.9\%) PTB patients had cough for <2 weeks. The remaining 106 (60.9\%) PTB patients reported 
cough for $\geqslant 2$ weeks. Thus, our strategy of screening any patient who could provide a sputum sample regardless of cough report or cough duration increased the detection of PTB from 106 cases to 174 cases during the study period, which translates into a $64.2 \%$ (95\% CI 45.7\%-85\%; p<0.01) increase in detection compared to the classic case finding definition of cough for $\geqslant 2$ weeks. The number needed to screen to detect one case of tuberculosis (NNS) was 5.4.

Susceptibility testing could be performed in 171 of the 174 isolates. Among these, there were $27(15.8 \%)$ MDR tuberculosis cases. Of these, five patients (18.5\%) reported no cough and two (7.4\%) reported cough for $<2$ weeks.

Using the classic definition of screening individuals with cough for $\geqslant 2$ weeks, only 442 persons would have been screened during the study period, and 106 (23\%) persons would have been found to have PTB. The NNS would have been 4.2. Therefore, our case finding strategy required screening 1.2 more persons for each case of PTB detection, compared to the classic case definition of cough for $\geqslant 2$ weeks.

We show that a simple tuberculosis case-finding strategy of obtaining a sputum sample for AFB smear and culture in any patient who is being evaluated in the emergency department for $\geqslant 2 \mathrm{~h}$ and is able to provide an adequate sputum specimen increased PTB case detection by $64.2 \%$, compared with the classic case-finding strategy of screening those with cough for $\geqslant 2$ weeks. Although the number of patients screened with our strategy was approximately twice the number of patients that would have been screened during the study period if only those with cough for $\geqslant 2$ weeks had been included, the significant increase in PTB case detection and the still relatively low NNS supports the potential utility of this strategy in emergency department settings with high tuberculosis prevalence.

Active screening for tuberculosis is recommended in high-prevalence settings [8]. Although emergency departments serve as the frontline for the sickest patients coming from the community and may be the main portal of entry to care for persons who otherwise would not seek routine medical care, few studies have focused on strategies for tuberculosis case finding in emergency departments. In a local emergency department in Porto Alegre, Brazil, where the rate of tuberculosis is $\sim 100$ cases per 100000 inhabitants, $15 \%$ of all comers who reported cough for $\geqslant 2$ weeks were found to have PTB, highlighting the potential utility of regularly screening persons who attend emergency departments for any reason in high tuberculosis prevalence settings [4].

One of the key components of the WHO framework for ending the global tuberculosis epidemic to eventually reach elimination is ensuring early detection of tuberculosis cases [9-11]. Our screening

\begin{tabular}{|c|c|c|c|c|c|}
\hline Characteristic & All PTB & No cough & Cough $<2$ weeks & Cough $\geqslant 2$ weeks & p-value \\
\hline Patients n & 174 & 42 & 26 & 106 & \\
\hline Age years & $30(22-44)$ & $34(24-52)$ & $25(20-35)$ & $31(22-42)$ & 0.03 \\
\hline Male sex & $125(71.8)$ & $30(71.4)$ & $18(69.2)$ & $77(72.6)$ & 0.94 \\
\hline Residence outside Lima & $4(2.3)$ & $1(2.4)$ & 1 (3.9) & $2(1.9)$ & 0.84 \\
\hline Unemployed & 70 (40.2) & $18(42.9)$ & 7 (26.9) & $45(42.5)$ & 0.32 \\
\hline Prior tuberculosis & $57(32.8)$ & $16(38.1)$ & 5 (19.2) & 36 (33.9) & 0.25 \\
\hline Tuberculosis contact & 33 (18.4) & $6(14.3)$ & 5 (19.2) & $21(19.8)$ & 0.66 \\
\hline Households & $4(3-6)$ & $4(2-6)$ & $3(3-6)$ & $4(3-6)$ & 0.15 \\
\hline Admission within a year & $24(13.8)$ & $6(14.3)$ & $3(11.5)$ & $15(14.2)$ & 0.94 \\
\hline Ever in prison & $10(5.8)$ & $4(9.5)$ & 0 & 6 (5.7) & 0.26 \\
\hline Subjective fever & $95(54.6)$ & $17(40.5)$ & $16(61.5)$ & $62(58.5)$ & 0.10 \\
\hline Weight loss & $131(75.3)$ & $30(71.4)$ & $15(57.7)$ & $86(81.1)$ & 0.04 \\
\hline Malaise & $118(67.8)$ & 27 (64.3) & $14(53.9)$ & $77(72.6)$ & 0.16 \\
\hline Dyspnoea & $87(50)$ & 14 (33.3) & 12 (46.2) & $61(57.6)$ & 0.03 \\
\hline Steroid use & $3(1.7)$ & 0 & $1(3.9)$ & $2(1.9)$ & 0.49 \\
\hline Diabetes & $4(2.3)$ & 0 & $1(3.9)$ & $3(2.8)$ & 0.48 \\
\hline MDR-TB & $27(15.8)$ & 5 (12.5) & $2(7.7)$ & $20(19.1)$ & 0.29 \\
\hline HIV infection ${ }^{+}$ & 30 (19.6) & $10(25.6)$ & $4(18.2)$ & $16(17.4)$ & 0.55 \\
\hline
\end{tabular}

Data are presented as $n(\%)$ or median (interquartile range), unless otherwise stated. MDR-TB: multidrugresistant tuberculosis. \# : p-value obtained from comparing no cough, cough $<2$ weeks and cough $\geqslant 2$ weeks groups using Kruskal-Wallis test for age and Chi-square test for all others. ": Mycobacterium tuberculosis susceptibility panel was available in 171 of 175 patients. ${ }^{+}$: HIV status was available in 153 of 174 patients. 
method is in line with the WHO efforts by recognising emergency departments as potentially useful settings for tuberculosis screening and by enhancing tuberculosis identification using this simple strategy.

Our study had limitations. We could not recruit patients during night shifts or Sundays due to resource constraints. This may have introduced selection bias. To account for the low sensitivity of a single sputum AFB smear, we also performed a sputum AFB culture. This could be a limitation as M. tuberculosis usually takes $\geqslant 2$ weeks to grow so linkage to tuberculosis care may be significantly delayed or not happen at all. Of note, $35 \%$ of PTB cases in this study had a negative AFB smear and would therefore potentially be lost to follow-up. The use of newer diagnostic technologies, such as the Xpert MTB/RIF assay (Cepheid, Sunnyvale, CA, USA), which is highly sensitive and has a rapid turnaround time may help overcome this limitation, in addition to providing information on rifampicin susceptibility [12].

Cough is a subjective symptom experienced differently by each person [13]. BREEN et al. [14] reported the absence of cough in $14 \%$ of PTB cases diagnosed at a tuberculosis clinic in the UK. Our study showed that almost a quarter of patients who were diagnosed with PTB reported no cough but were able to provide a sputum sample. There is increasing interest in understanding the mechanisms of cough and reliability of cough assessment tools in tuberculosis patients [15]. In the meantime, assessing the ability to provide a sputum sample for AFB testing in addition to asking for the presence of cough may be useful in emergency departments with high tuberculosis prevalence, such as ours, in order to enhance tuberculosis case finding. Further studies are required to validate our findings in similar settings.

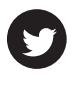

$@$ ERSpublications

Screening all comers able to provide a sputum sample increased TB detection in an emergency department in Peru http://ow.ly/T7pHA

Eduardo Ticona ${ }^{1,2}$, Moises A. Huaman ${ }^{3}$, Luz Maria Huaroto ${ }^{1,2}$, Marcos Burgos ${ }^{4}$, Meghan Brett ${ }^{4}$, Rod Escombe ${ }^{5}$ and David A.J. Moore ${ }^{5}$

${ }^{1}$ Infectious Diseases and Tropical Medicine Service, Dept of Medical Specialties, Hospital Nacional Dos de Mayo, Lima, Peru. ${ }^{2}$ Dept of Medicine, Universidad Nacional Mayor de San Marcos, Lima, Peru. ${ }^{3}$ Division of Infectious Diseases, Dept of Medicine, University of Kentucky, Lexington, KY, USA. ${ }^{4}$ Division of Infectious Diseases, Dept of Medicine, University of New Mexico, Albuquerque, NM, USA. ${ }^{5}$ TB Centre and Department of Clinical Research, London School of Hygiene and Tropical Medicine, London, UK.

Correspondence: Moises A. Huaman, Division of Infectious Diseases, University of Kentucky, 740 South Limestone, K512, Lexington, KY 40536. E-mail: moises.huaman@uky.edu

Received: June 032015 | Accepted after revision: Sept 102015 | First published online: Oct 222015

Support statement: M.A. Huaman received support from the National Center for Research Resources and the National Center for Advancing Translational Sciences, National Institutes of Health (NIH), through Grant UL1TR000117. The content is solely the responsibility of the authors and does not necessarily represent the official views of the NIH. David AJ Moore received support from the Wellcome Trust, through Grant 078067/Z/05/Z. Funding information for this article has been deposited with FundRef.

Conflict of interest: None declared.

\section{References}

1 World Health Organization. The Global Plan to Stop TB 2011-2015. Geneva, Switzerland, 2010.

2 Dheda K, Gumbo T, Gandhi NR, et al. Global control of tuberculosis: from extensively drug-resistant to untreatable tuberculosis. Lancet Respir Med 2014; 2: 321-338.

3 World Health Organization. An expanded DOTS framework for effective tuberculosis control. Geneva, Switzerland, 2002.

4 Silva DR, Muller AM, Tomasini Kda S, et al. Active case finding of tuberculosis (TB) in an emergency room in a region with high prevalence of TB in Brazil. PLoS One 2014; 9: e107576.

5 Oficina de Estadística y Programa de Control de la Tuberculosis DISA V Lima Ciudad. Ministerio de Salud, Peru, 2006.

6 World Health Organization. Laboratory services in tuberculosis control. Part III. Culture. Geneva, Switzerland, 1999.

7 Canetti G, Rist N, Grosset J. [Measurement of sensitivity of the tuberculous bacillus to antibacillary drugs by the method of proportions. Methodology, resistance criteria, results and interpretation.] Rev Tuberc Pneumol (Paris) 1963; 27: 217-272.

8 Lonnroth K, Corbett E, Golub J, et al. Systematic screening for active tuberculosis: rationale, definitions and key considerations. Int J Tuberc Lung Dis 2013; 17: 289-298.

9 World Health Organization. Draft global strategy and targets for tuberculosis prevention, care and control after 2015. Report by the Secretariat. Sixty-seventh World Health Assembly document A67/11 2014. Available from http://apps.who.int/gb/ebwha/pdf_files/WHA67/A67_11-en.pdf Date last accessed: September 6, 2015.

10 D'Ambrosio L, Dara M, Tadolini M, et al. Tuberculosis elimination: theory and practice in Europe. Eur Respir J 2014; 43: 1410-1420.

11 Lonnroth K, Migliori GB, Abubakar I, et al. Towards tuberculosis elimination: an action framework for low-incidence countries. Eur Respir J 2015; 45: 928-952. 
12 Boehme CC, Nicol MP, Nabeta P, et al. Feasibility, diagnostic accuracy, and effectiveness of decentralised use of the Xpert MTB/RIF test for diagnosis of tuberculosis and multidrug resistance: a multicentre implementation study. Lancet 2011; 377: 1495-1505.

13 Schmit KM, Coeytaux RR, Goode AP, et al. Evaluating cough assessment tools: a systematic review. Chest 2013; 144: $1819-1826$

14 Breen RA, Leonard O, Perrin FM, et al. How good are systemic symptoms and blood inflammatory markers at detecting individuals with tuberculosis? Int J Tuberc Lung Dis 2008; 12: 44-49.

15 Turner RD, Bothamley GH. Cough and the Transmission of Tuberculosis. J Infect Dis 2015; 211 : 1367-1372.

\section{Ertapenem in the treatment of multidrug-resistant tuberculosis: first clinical experience}

To the Editor:

Treatment of multidrug-resistant (MDR) tuberculosis (TB) (defined as resistance to at least isoniazid and rifampicin, two core first-line drugs for TB treatment) and extensively drug-resistant (XDR)-TB (defined as resistance to isoniazid and rifampicin plus any fluoroquinolone and at least one of the injectable drugs amikacin, capreomycin or kanamycin) are a challenge for both clinicians and public health specialists [1-5]. Treatment outcomes of difficult-to-treat MDR-TB cases (e.g. those with an extensive resistance pattern) are still unsatisfactory, adverse events frequent and severe, and the necessary drugs expensive [6].

A previous proof-of-concept study from our group has suggested that meropenem clavulanate is potentially useful in the treatment of these cases and is also well tolerated [7]. In vitro and pharmacological data suggest that ertapenem, a newer carbapenem compound, is potentially active against Mycobacterium tuberculosis, its longer half-life allowing single daily parenteral administration (rather than thrice-daily administration of meropenem) and advantageous homecare or day-hospital management $[8,9]$.

Clinical information necessary to study safety, tolerability and efficacy of ertapenem was retrospectively collected on all MDR-TB cases treated at the E. Morelli Hospital (the TB reference centre for Italy) between 2008 and 2015. Carbapenems were used in MDR-TB patients lacking active drugs in their regimen. Meropenem ( $3 \mathrm{~g}$ three times daily) or imipenem (500 $\mathrm{mg}$ four times a day) was given during hospital stay and if limited drug options switched to ertapenem ( $1 \mathrm{~g}$ once daily) for easier home-care or day-hospital administration.

Regimens to treat MDR/XDR-TB cases were tailored to drug susceptibility testing (DST) results according to World Health Organization (WHO) recommendations [2] to design an Optimised Background Regimen (OBR), using fluoroquinolones, injectable agents and other second-line oral agents co-administered with linezolid and carbapenems in heavily resistant/drug-intolerant individuals. A team composed of three external investigators reviewed and agreed on each clinical record reporting on the use of carbapenems in the study period, in collaboration with local specialists.

The database was developed to be consistent with that used in previous studies $[7,10,11]$. The protocol and outcome definitions were compatible with those used to study the largest MDR-TB cohort available [3, 4].

A patient who completed treatment as recommended by the national policy without evidence of failure and was consistently culture-negative with at least three or more consecutive cultures taken at least 30 days apart after the intensive phase was defined as cured. Treatment failure was defined as treatment terminated or need for a permanent regimen change of at least two anti-TB drugs because of: 1) lack of conversion by the end of the intensive phase; 2 ) bacteriological reversion in the continuation phase after conversion to negative; 3 ) evidence of additional acquired resistance to fluoroquinolones or second-line injectable drugs; or 4) adverse drug reactions [1].

Sputum conversion was defined as two consecutive negative sputum smears in patients who were sputum smear-positive at diagnosis. Time to culture conversion was defined as time from treatment start to the date of the first of two consecutive negative cultures $[7,10]$. 